\title{
Psychosocial Stress-Induced Analgesia: An Examination of Effects on Heat Pain Threshold and Tolerance and of Neuroendocrine Mediation
}

\author{
Jens Gaab ${ }^{a}$ Julia Jiménez ${ }^{a} \quad$ Livia Voneschen $^{a} \quad$ Daniel Oschwald ${ }^{a}$ \\ Andrea H. Meyer ${ }^{b}$ Urs M. Nater ${ }^{\mathrm{e}}$ Peter Krummenacher ${ }^{\mathrm{a}, \mathrm{c}, \mathrm{d}}$ \\ Divisions of a Clinical Psychology and Psychotherapy and ${ }^{b}$ Clinical Psychology and Epidemiology, Department of \\ Psychology, University of Basel, Basel, and 'Brainability LLC, and ${ }^{\mathrm{d}}$ Collegium Helveticum, University of Zurich and \\ ETH Zurich, Zurich, Switzerland; ${ }^{e}$ Division of Clinical Biopsychology, Department of Psychology, Philipps University \\ Marburg, Marburg, Germany
}

\section{Keywords \\ Stress-induced analgesia $\cdot$ Psychosocial stress $\cdot$ Cortisol}

\begin{abstract}
Stress-induced analgesia ( $\mathrm{SIA}$ ) is an adaptive response of reduced nociception following demanding acute internal and external stressors. Although a psychobiological understanding of this phenomenon is of importance for stress-related psychiatric and pain conditions, comparably little is known about the psychobiological mechanisms of SIA in humans. The aim of this study was to investigate the effects of acute psychosocial stress on heat pain perception and its possible neuroendocrine mediation by salivary cortisol levels and a-amylase activity in healthy men. Employing an intra-individual assessment of heat pain parameters, acute psychosocial stress did not influence heat pain threshold but significantly, albeit slightly, increased heat pain tolerance. Using linear mixed-model analysis, this effect of psychosocial stress on heat pain tolerance was not mediated by increases of salivary cortisol and state anxiety levels or by the activity of a-amylase. These results show that while psychosocial stress
\end{abstract}

\section{KARGER}

(c) 2017 S. Karger AG, Basel

E-Mail karger@karger.com

www.karger.com/nps is selectively analgesic for heat pain tolerance, this observed effect is not mediated by stress-induced increases of salivary cortisol and a-amylase activity, as proxies of both the hypothalamus-pituitary-adrenal axis and the autonomic nervous system activation.

(c) 2017 S. Karger AG, Basel

\section{Introduction}

Stress-induced analgesia (SIA) can be conceptualized as an adaptive nociceptive response following exposure to a conditioned or unconditioned stressful or fearful stimulus. It has been widely reported in animals $[1,2]$ and to a lesser and more heterogeneous extent in humans [for examples, see 3-9]. In humans, SIA has been elicited using physical [10], psychological [7, 11], and psychosocial [6] stressors. Whether an aversive stimulus induces analgesia or hyperalgesia is influenced by several factors such as the valence, type of emotional response, and magnitude of the arousal [12-18] as well as its predictability [19]. 
SIA is a complex process that is mediated by the activation of descending inhibitory pain pathways. It is modulated by opioid $[9,20]$ and non-opioid mechanisms involving monoamines $[21,22]$ as well as endocrine processes [23] involved in the regulation of stress. While the involvement of the corticotropin-releasing factor as well as glucorticosteroids has been found to be important in the development of SIA in animals [23-29], comparable endocrine evidence in humans is scarce. On the one hand negative associations between cortisol and pain perception have been reported with regard to cortisol and pain responses to the cold pressor test as well as to noxious thermal stimulations $[9,30]$. On the other hand, cortisol responses did not affect the association between the effects of stress and pain rating in the cold pressor test [6].

The autonomic nervous system (ANS) interacts with nociceptive processing in a complex manner at all levels of the neuraxis [31] since afferent sympathetic fibres process sensory information from nociceptors [32]. In animals, a sympathetic-adrenal-medullary-induced increase of catecholamines has been associated with elevated cardiac and vascular activity, which is linked with diminished pain sensitivity in animals [33] and in male humans $[11,34,35]$. Moreover, hypothalamic-autonomic activation associated with systolic blood pressure level has been suggested as one likely mechanism underlying SIA [6]. Also, increased salivary a-amylase (sAA) activity has been shown during the cold pressure test [36] and was recently suggested as an indirect physiological correlate of subjective heat pain perception [37]. However, to our knowledge, no studies have investigated whether and to what extent sAA, as a marker of ANS activity, influences the relationship between psychosocial stress and heat pain perception.

Therefore, we set out to investigate the effects of acute psychosocial stress on heat pain perception and its possible neuroendocrine mediation by salivary cortisol levels and sAA activity in healthy men using linear mixed-model analysis. Employing a standardized psychosocial stress test paradigm - the Trier social stress test (TSST) [38] and a heat-pain paradigm [39], we tested the following hypotheses. First, we predicted that acute psychosocial stress exposure reduces the nociceptive response (SIA) to heat pain stimuli reflected by higher pain threshold and tolerance. Second, we examined whether that SIA is mediated by cognitive stress appraisal - affective or neuroendocrine responses to stress as evidenced by higher levels of both parameters after the TSST and by a positive association of these parameters with pain threshold and tolerance.

\section{Methods}

\section{Participants}

Right-handed men aged between 18 and 40 years were recruited by e-mail or oral promotion in lectures of the Department of Psychology, University of Basel. The e-mail contained a link to an internet site with basic information about the study titled "Influence of psychosocial stress on pain perception" and a questionnaire to assess the following exclusion criteria: left-handedness [40], female gender [41], smoking [42], alcohol consumption (regularly and more than 3 units per day, 1 unit $\triangleq 8$ g ethanol) [43], drug use, and any acute or chronic somatic or psychiatric disorder [44-46]. Participants had to be naive to the employed heat pain measurement (TSA; Medoc, Ramat-Yishai, Israel) and stress test (TSST) to avoid any confounding effects. For compensation, participants received university credits for their bachelor in psychology studies or CHF 100. All subjects were provided with complete written and oral descriptions of the study and gave written consent before the assessment started. The study was approved by the cantonal ethics committee.

\section{Experimental Conditions}

Eligible participants were randomly allocated to either the stress condition first followed by a control condition 1 week later $(n=15)$ or vice versa $(n=14)$.

In the stress condition, the participants were subjected to the TSST, which has repeatedly been found to induce strong endocrine and cardiovascular responses in $70-80 \%$ of the subjects tested [38, 47]. After a baseline heat pain measurement, the participants were introduced to the TSST (Intro, $2 \mathrm{~min}$ ). They were then returned to a room, where they had $10 \mathrm{~min}$ on their own to prepare and complete a questionnaire designed to assess cognitive appraisal processes (Pennsylvania Alternate System of Assessment, PASA) [48] regarding the anticipated stress situation. Afterwards, the subjects were taken back into the TSST room, where they took part in a simulated job interview ( $5 \mathrm{~min}$ ) followed by a mental arithmetic task ( $5 \mathrm{~min})$ in front of an audience of 2 people. Five minutes after the TSST, the participants underwent the second heat pain measurement. The heat pain measurements and the TSST were conducted in separate rooms. Before and after the TSST as well as in the control condition, the participants stayed in a room on their own, except for heat pain measurements (see below). Salivary samples were repeatedly assessed in both conditions before and after the TSST $(-60,-30,-15,0,+10,+20,+30,+45$, and $+60 \mathrm{~min}$, with 0 min assessed directly after the TSST). In the control condition, the participants stayed in a quiet room on their own for the time of the assessments.

\section{Sampling Methods and Biochemical Analyses}

Saliva was collected from the subjects using Salivette collection devices (Sarstedt, Sevelen, Switzerland). Sampling time was exactly $1 \mathrm{~min}$, during which subjects had to chew on the cotton swabs as regularly as possible. Salivettes were stored at $-20^{\circ} \mathrm{C}$ after completion of the session until biochemical analysis took place. To assess salivary cortisol responses, salivary cortisol levels in all 9 salivette samples were analysed; to assess responses of the activity of sAA, the first 5 salivettes were used for biochemical analyses.

After thawing, the following biochemical analyses were conducted in the biochemical laboratory of the Clinical Psychology and Psychotherapy Department at the University of Zurich, Swit- 
zerland: for salivary cortisol analyses a highly sensitive liquid chromatography-tandem mass spectrometry (LC-MS/MS) method was used [49], and the activity of sAA was analysed with a kinetic colorimetric test using assay kits (Roche 11555685 a-Amylase Liquid acc.) and the automatic analyser (Biotek Instruments, Lucerne, Switzerland) with software KC4 (Roche, Basel, Switzerland).

\section{Heat Pain Stimuli and Measurement}

Heat pain measurements were performed 4 times per participant, thus before and after each experimental condition (TSST or control condition). Heat stimuli were administered in counterbalanced order to the left and right volar forearm using a $30 \times 30 \mathrm{~mm}$ Peltier device (TSA-II; Medoc) placed at two-thirds of the distance from wrist to elbow. Individual pain threshold was measured using the search method starting at $42^{\circ} \mathrm{C}$ : the participants were asked to increase or decrease the magnitude of the heat stimulus to the transition point where they felt it changing from "hot" to "painful." The participants were instructed to determine the transition point as precisely as possible and to use equally long response intervals (i.e., mouse click intervals) for altering temperature, and also to apply combined bottom-up (increasing temperature) and topdown (decreasing temperature) search strategies.

Pain tolerance was determined by the method of limits: the participants were asked to stop the increasing heat stimulus at the moment they could no longer stand it. Three measurements starting at $35^{\circ} \mathrm{C}$, with a rise of $0.8^{\circ} \mathrm{C} / \mathrm{s}$, were averaged. To avoid physical injury, the pain tolerance measurement stopped automatically at $52^{\circ} \mathrm{C}$. Prior to the actual measurements, the participants were familiarized with the heat stimuli and the controlling device. Pain threshold was always measured prior to pain tolerance in order to minimize interactions between pain threshold and pain tolerance [39]. All participants started with a practice trial for threshold and tolerance, counterbalanced for left and right forearm sites.

Subjective pain intensity and valence rating were performed each time after pain tolerance measurement. The subjects were asked to rate the perceived intensity of the just-perceived heat pain stimulus in a computerized scroll bar visual analogue scale in the range of 1-100, where 1 was the lowest and 100 the highest everexperienced pain. For pain valence, 1 represented a neutral sensation and 100 an extremely unpleasant sensation.

\section{Statistical Analyses}

Primary outcomes were the within-subject difference scores (in ${ }^{\circ} \mathrm{C}$ ) of heat pain threshold and tolerance between the 2 measurements (before and after TSST or control condition). Negative values reflected relative hyperalgesia, while positive values indicated relative analgesia. Cortisol as well as anxiety responses over time in both conditions were treated as secondary outcomes. MANCOVAs and MANOVAs for repeated measures were computed to analyse primary and secondary outcomes, controlling for covariates as indicated. Subsequent ANOVA or ANCOVA for repeated measures were corrected by the Greenhouse-Geisser procedure when the sphericity assumption was violated. KolmogorovSmirnov tests showed that sAA activity data were not normally distributed. Calculating the log of sAA activity values produced normally distributed values. The log-transformed cortisol values were used for all statistical analyses, but means and standard deviations of untransformed values are presented. Data were also tested for homogeneity of variance using the Levene test before statistical procedures were applied. The optimal total sample size of $N=30$ to detect an expected medium effect size of $\mathrm{f}=0.25$, with a $90 \%$ power and $\alpha=0.05$, was calculated a priori with the statistical software G-Power (version 3.1 [50]).

To assess associations between psychological and cortisol parameters, bivariate (Pearson product-moment and partial correlations) and multivariate (hierarchical multiple regression analysis) methods were used. For salivary cortisol and $\alpha$-amylase parameters, areas under the response curve were calculated with respect to increase (AUCi) and with respect to ground (AUCg) using the trapezoidal method as an indicator for integrated responses in the TSST [51]. For all analyses, the significance level was 5\%. Estimates of explained variance for significant repeated measures are reported in Cohen's $\mathrm{f}$ (calculated from SPSS partial eta-squared with the program $G^{*}$ Power 3.1, where $0.1=$ small, $0.25=$ medium, and $0.45=$ large).

We used a linear mixed model [52] with condition (TSST vs. control) and the mediators AUCg for salivary cortisol and AUCg for $\alpha$-amylase as time-varying covariates [53] since both can vary within subjects (lower-level mediation model). Outcome variables were the difference scores (before and after TSST or control condition) of pain tolerance and pain threshold. Separate analyses were set up for each combination of outcome and mediator ( 4 analyses altogether). In order to estimate total, direct, and indirect (i.e., mediated) effects, 3 models were set up [54]:

Model 1: $Y_{i j}=\zeta_{0}+c C O N D_{i j}+u_{0 i}+\varepsilon_{i j}$

Model 2: $M E D_{i j}=\zeta_{1}+a C O N D_{i j}+u_{1 i}+\varepsilon_{i j}$

Model 3: $Y_{i j}=\zeta_{2}+c^{\prime} C O N D_{i j}+b M E D_{i j+}+u_{2 i}+\varepsilon_{i j}$

$Y_{i j}, C O N D_{i j}$, and $M E D_{i j}$ denote the observed values of the outcome (tolerance or threshold), condition (TSST vs. control), and mediator (salivary cortisol or a-amylase), respectively, of person $i$ at time point $j ; \zeta_{0}$ to $\zeta_{2}$ denote the intercept, $u_{0 i}$ to $u_{2 i}$ the personspecific intercept (deviation from/to $\zeta_{2}$ ), and $\varepsilon_{i j}$ the error term. Non-mediated Model 1 tests for the effect of condition on the respective outcome. Model 2 tests for the effect of condition on the respective mediator (coefficient $a$ ). Model 3 tests for the effect of condition on the outcome (coefficient $c^{\prime}$ ) in the presence of the mediator (coefficient $b$ ). All 3 models allow each participant to have their own intercept $\left(u_{0 i}\right.$ to $\left.u_{2 i}\right)$.

The following steps are required to establish mediation in the present study [55]: (1) the outcome is affected by the predictor ( $c$ significantly differs from 0$)$; (2) the mediator is affected by the predictor ( $a$ significantly differs from 0$)^{1}$; $(3)$ the outcome is still affected by the mediator after controlling for the predictor ( $b$ significantly differs from 0 ); (4a) complete mediation: the outcome is no longer affected by the predictor after controlling for the mediator ( $c^{\prime}$ does not significantly differ from 0$)$; (4b) partial mediation: the outcome is less affected by the predictor after controlling for the mediator than when not controlling for it ( $c^{\prime}$ significantly differs from 0 but is less than $c$ ). Total effects are represented by $c$, direct effects by $c^{\prime}$, and mediated effects by the product $a b$. In addition, the proportion of the total mediated effect is presented. To obtain standard errors of mediated effects we used equations 5.7 [56]. Mediation effects were tested for statistical significance by dividing estimates by their standard error, assuming normal distribution of results. Analyses were carried out using SPSS, rel. 14.0 and for the mediation analysis Mplus, rel. 6.

\footnotetext{
1 If either $a_{1}$ or $a_{2}$ is not significantly smaller than 0 then the corresponding mediating variable is not a mediator.
} 


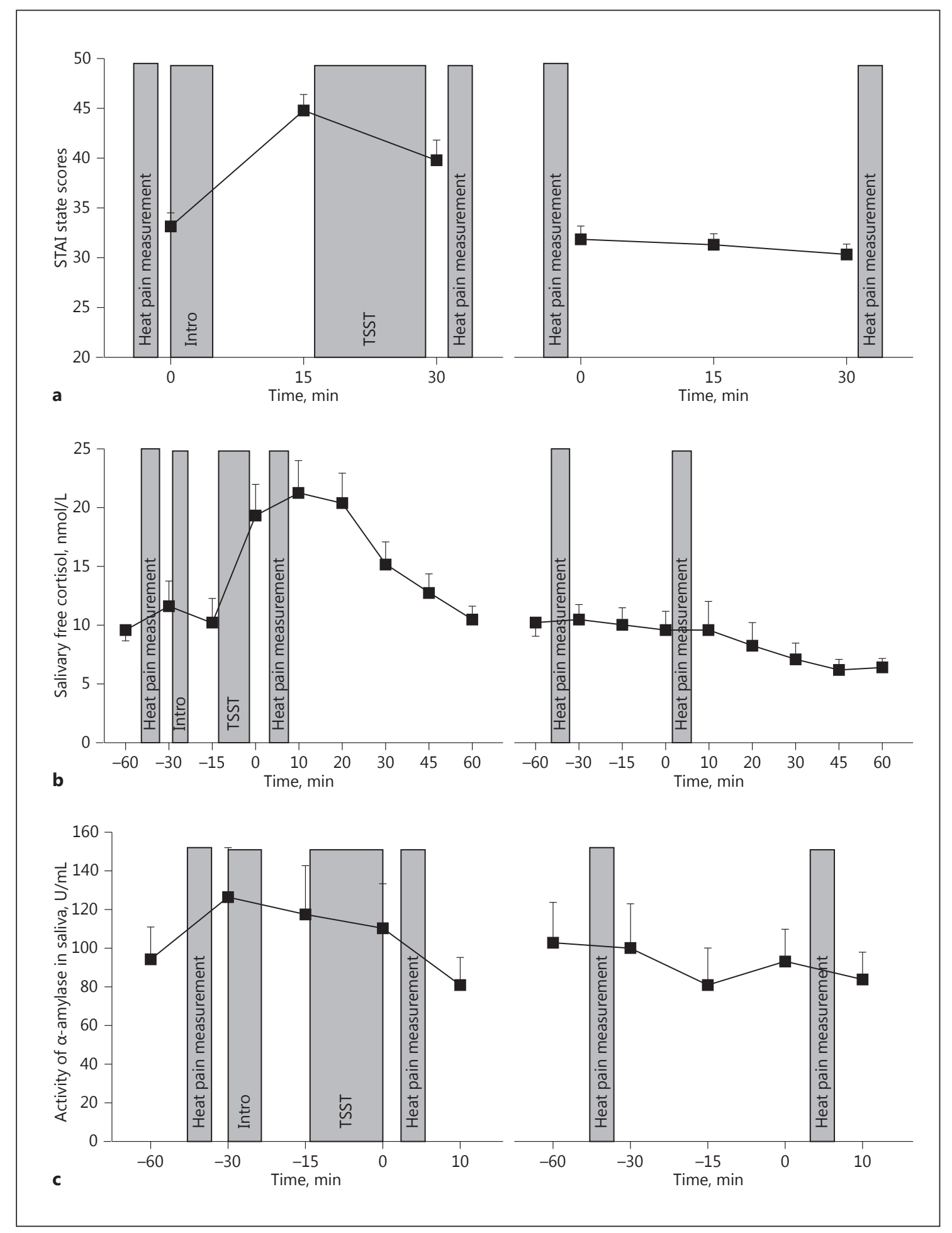

Fig. 1. Changes of STAI state scores (a), salivary free cortisol (b) and activity of $a$-amylase in saliva over time (c) in the TSST (left) and in the control condition (right). 


\section{Results}

\section{Participants}

Overall, 29 healthy male subjects participated in the study (mean age in years: 24.6, range 18-34; mean BMI, 22.9, SD 2.7). All participants completed both the TSST and the control condition. The mean State-Trait Anxiety Inventory (STAI) trait anxiety T scores of the participants were slightly, but not significantly, higher than published norms (normative $\mathrm{T}$ score $=50$, population mean $=53.6$; $\left.\mathrm{t}_{26}=1.8, p=0.07\right)$.

\section{Subjective and Biological Stress Responses}

Participants differed in their state anxiety levels over time between the TSST and the control condition $(\mathrm{F}(4$, $112)=9.0, p<0.001, \mathrm{f}=0.56)$, with a strong increase in the $\operatorname{TSST}(\mathrm{F}(1.7,48.5)=17.5, p<0.001, \mathrm{f}=0.78)$ and a decrease in the control condition $(\mathrm{F}(1.5,43.1)=3.5, p=0.05, \mathrm{f}=$ 0.35; see Fig. 1a). Also, cognitive appraisal differed significantly between the respective conditions $(\mathrm{F}(4,25)=12.2$, $p<0.001, \mathrm{f}=1.39$ ), with higher stress appraisal before the TSST in comparison with the control condition. Salivary cortisol levels differed between the TSST and the control condition $(\mathrm{F}(16,448)=10.00, p<0.001, \mathrm{f}=0.73)$, with robust increases of salivary cortisol levels in the $\operatorname{TSST}(\mathrm{F}(2.6$, $73.5)=19.5, p<0.001, \mathrm{f}=0.83)$ and decreases in the control condition $(\mathrm{F}(1.6,44.1)=3.6, p<0.04, \mathrm{f}=0.37$; see Fig. $1 \mathrm{~b})$. Values for the AUC differed significantly between conditions for salivary cortisol $(\mathrm{F}(2,27)=44.6, p<0.001, \mathrm{f}=1.83$; AUCg: $\mathrm{F}(1,28)=85.0, p<0.001, \mathrm{f}=1.73$; AUCi: $\mathrm{F}(1$, $28)=40.6, p<0.001, \mathrm{f}=1.2)$. With regard to the activity of sAA, a significant time by condition effect was observed $(\mathrm{F}(4,25)=2.8, p=0.048, \mathrm{f}=0.67)$, with significant increases in the TSST condition $(\mathrm{F}(4,25)=3.5, p=0.02, \mathrm{f}=0.75)$ but no significant changes over time in the control condition $(\mathrm{F}(4,25)=0.7, p=0.63$; see Fig. $1 \mathrm{c})$. A similar pattern was observed for integrated responses of sAA, with significant differences between condition for AUCi but not for $\operatorname{AUCg}(\mathrm{F}(2,27)=2.9, p=0.07$; $\operatorname{AUCg}: \mathrm{F}(1,28)=1.2$, $p=0.28$; AUCi: $\mathrm{F}(1,28)=5.9, p=0.02, \mathrm{f}=0.45)$.

\section{Pain Threshold and Tolerance}

Repeated multivariate analyses indicated a significant pre- to post-assessment effect for the TSST condition $(\mathrm{F}(2,27)=3.5, p=0.04, \mathrm{f}=0.52)$. Subsequent univariate analyses showed that this effect was driven by a pre-post increases in heat pain tolerance $(\mathrm{F}(1,28)=7.3, p=0.01$, $\mathrm{f}=0.52)$ but not in heat threshold $(\mathrm{F}(1,28)=0.002, p=$ $0.96)$, Also, there were no pre-post changes in the control condition $(\mathrm{F}(2,27)=0.3, p=0.97$; heat pain tolerance $\mathrm{F}(1$,

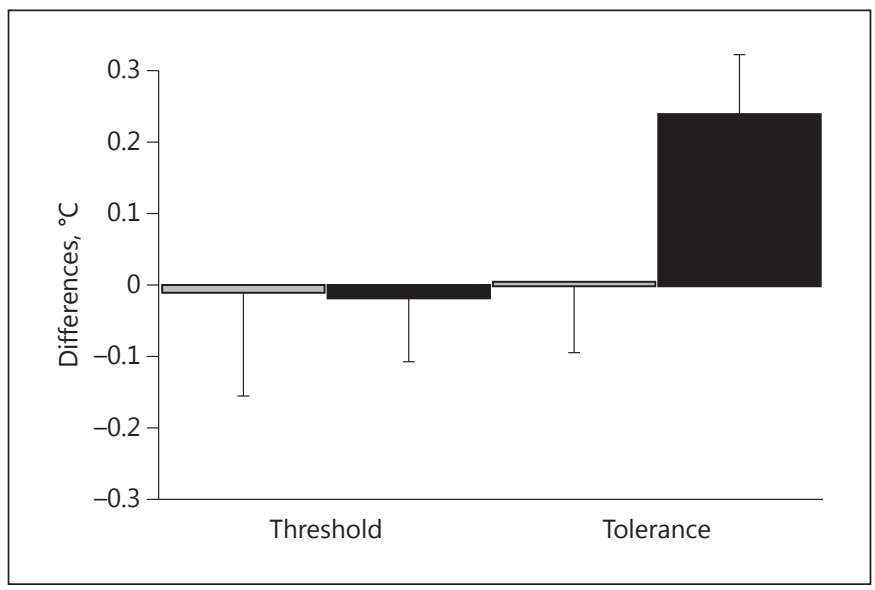

Fig. 2. Within-subject difference scores in heat pain threshold and tolerance for the control (grey bars) and the TSST condition (black bars).

Table 1. Pre- and post-threshold and tolerance levels and subjective pain ratings in the control and the TSST condition

\begin{tabular}{llllll}
\hline & \multicolumn{3}{l}{ Control } & & \multicolumn{2}{l}{ TSST } \\
\cline { 2 - 3 } \cline { 6 - 7 } & Pre & Post & & Pre & Post \\
\hline Threshold, ${ }^{\circ} \mathrm{C}$ & $46.5 \pm 1.5$ & $46.5 \pm 1.6$ & & $46.5 \pm 1.4$ & $46.5 \pm 1.8$ \\
Tolerance, ${ }^{\circ} \mathrm{C}$ & $49.8 \pm 1.1$ & $49.8 \pm 1.1$ & & $49.7 \pm 1.1$ & $50.0 \pm 1.1$ \\
Pain valence & $69.4 \pm 3.9$ & $69.1 \pm 3.8$ & & $69.4 \pm 4.3$ & $69.4 \pm 4.1$ \\
Pain intensity & $63.9 \pm 3.4$ & $63.8 \pm 3.0$ & & $65.4 \pm 3.4$ & $66.7 \pm 3.2$ \\
\hline
\end{tabular}

28) $=0.00, p=0.99$; heat threshold $\mathrm{F}(1,28)=0.05, p=$ 0.82) (see Table 1).

Within-subject difference scores in heat pain threshold and tolerance differed significantly between conditions $(\mathrm{F}(2,27)=3.4, p=0.05, \mathrm{f}=0.5)$, with significant differences between condition for heat pain tolerance $(\mathrm{F}(1,28)=5.8, p=0.02, \mathrm{f}=0.45)$ but not for heat pain threshold $(\mathrm{F}(1,28)=0.003, p=0.96$; see Fig. 2). Also, between-condition differences of within-subject (i.e., pre-post) differences in heat pain threshold and tolerance differed significantly $(\mathrm{F}(2,27)=3.37, p=0.05, \mathrm{f}=0.5)$, with no $\left(0.0^{\circ} \mathrm{C}\right)$ between-condition difference for heat pain threshold and a $0.26^{\circ} \mathrm{C}$ increase for heat pain tolerance, respectively.

\section{Subjective Pain Ratings}

Repeated multivariate analyses of pre- to post-assessment differences in valence and intensity ratings indicated significant differences between the TSST and control 
Table 2. Direct, total, and mediated effects of condition (TSST vs. control) on tolerance and threshold pain with mediators AUCg salivary cortisol and AUCg a-amylase

\begin{tabular}{|c|c|c|c|c|}
\hline & \multicolumn{2}{|l|}{ Tolerance } & \multicolumn{2}{|l|}{ Threshold } \\
\hline & salivary cortisol & $\alpha$-amylase & salivary cortisol & a-amylase \\
\hline$a$ & $0.81(0.22)^{* * *}$ & $1.24(1.11)$ & $0.81(0.22)^{* * *}$ & $1.24(1.11)$ \\
\hline$b$ & $6.15(6.40)$ & $0.56(0.90)$ & $13.24(9.24)$ & $1.62(1.29)$ \\
\hline$a b$ & $4.99(5.36)$ & $-0.69(0.013)$ & $10.74(8.04)$ & $2.02(2.42)$ \\
\hline Mediated proportion, $\%$ & 19 & - & - & - \\
\hline$c$ & $25.75(10.55)^{*}$ & $25.75(10.55)^{*}$ & $-0.862(16.86)$ & $-0.862(16.86)$ \\
\hline$c^{\prime}$ & $20.76(11.52)$ & $26.44(10.76)^{*}$ & $-11.61(17.92)$ & $-2.88(15.85)$ \\
\hline
\end{tabular}

Figures in parentheses indicate standard errors. Mediated proportion: where no results are indicated, the direct effect was higher than the indirect effect and the mediated proportion therefore not computed. $c$, total effect between the predictor condition (TSST vs. control) and the outcomes heat pain tolerance or heat pain threshold; $c^{\prime}$, direct effect between the predictor condition (TSST vs. control) and the outcomes heat pain tolerance or heat pain threshold; $a b$, indirect effect between predictor and outcome via salivary cortisol or $\alpha$-amylase, with mediated proportion defined as $a b / c .{ }^{*} p<0.05,{ }^{* * *} p<0.001$ (all other values were non-significant).

condition $(\mathrm{F}(2,27)=8.2, p=0.002, \mathrm{f}=0.78)$, with significant increases in intensity ratings in the TSST in comparison with the control condition $(\mathrm{F}(1,28)=15.0, p=$ $0.001, \mathrm{f}=0.73)$, whereas no significant pre-post differences in valence rating between condition were observable $(\mathrm{F}(1,28)=0.01, p=0.92$; see Table 1$)$.

\section{Associations between Stress Responses and Heat Pain \\ Threshold and Tolerance}

Correlations between integrated AUCg and AUCi salivary free cortisol, $\alpha$-amylase and cognitive anticipatory appraisal, as well as STAI state anxiety responses were non-significant in both conditions (all $r>0.20$, all nonsignificant). Accordingly, integrated STAI state anxiety (AUCg) and PASA score responses did not predict integrated salivary free cortisol or $\alpha$-amylase responses in both conditions (all non-significant, data not shown).

To analyse the associations between the integrated stress responses and pain parameters, hierarchical regression analyses were carried out with AUCg and AUCi of salivary free cortisol, $\alpha$-amylase, and STAI state anxiety responses as independent variables and heat pain threshold and tolerance parameters as dependent variables.

In both conditions, integrated biological and affective stress responses were unrelated to pain threshold (control condition: ${ }_{\text {adjusted }} R^{2}=-0.02 ; \mathrm{F}(6,22)=0.9, p=0.50$; TSST condition: $\left.{ }_{\text {adjusted }} R^{2}=0.003 ; \mathrm{F}(6,22)=1.0, p=0.44\right)$ and tolerance (control condition: adjusted $R^{2}=-0.08 ; \mathrm{F}(6,22)=$ $0.7, p=0.68$; TSST condition: adjusted $R^{2}=-0.24 ; \mathrm{F}(6,22)=$ $0.1, p=1.0$ ), with none of the regression coefficients
(AUCi and AUCg of salivary free cortisol, activity of sAA, and STAI state changes over time in the control and TSST condition) reaching significance (data not shown, all non-significant).

\section{Examination of Possible Mediation by Salivary \\ Cortisol and $\alpha$-Amylase}

Difference scores of heat pain tolerance were significantly increased in the TSST condition compared with the control condition (coefficient $c$ in Table 2, first and second columns). Also, AUCg of salivary cortisol was higher in the TSST relative to the control condition (coefficient $a$ ). However, since there was no association between AUCg of salivary cortisol values and pain tolerance (coefficient $b$ ), there was no significant mediation effect (coefficient $a b$ ), and only $19 \%$ of the total effect was mediated by AUCg of salivary cortisol (coefficient $a b /$ coefficient $c$ ). For the AUCg of sAA none of the relevant coefficients ( $a$, $b, a b)$ were significantly different from 0 , and as a consequence there was no mediation effect either (Table 2, second column). In addition, the direct effect of the TSST condition on pain tolerance (coefficient $c^{\prime}$ ) was still significant when controlling for the mediator AUCg of sAA and was very similar to the total effect (compare coefficients $c$ with $c^{\prime}$ in Table 2, second column).

As for heat pain tolerance, there was no mediation effect of the TSST condition on difference scores of heat pain threshold via mediators AUCg of salivary cortisol or AUCg of salivary amylase. There was also no total or direct effect of the TSST condition on pain threshold (coef- 
ficients $c$ and $c^{\prime}$, third and fourth columns in Table 2). Note that the direct effects when controlling for either mediator were of opposite sign relative to their respective indirect effects, suggesting inconsistent mediation. Inconsistent mediation is present when the direct and mediated effect of a predictor variable on an outcome variable has opposite signs, as contrasted with consistent mediation models in which the direct and mediated effects have the same sign [57]. Because direct and indirect effects were of fairly similar magnitudes and opposite signs, they cancelled each other out [58]. On the other hand, neither direct nor indirect effects were significant, and therefore this issue should not be overinterpreted.

\section{Discussion}

We set out to examine the effects of social-evaluative stress on heat pain threshold and tolerance and its possible mediation by hypothalamus-pituitary-adrenal (HPA) system-associated salivary cortisol levels and ANS-related sAA activity in healthy subjects employing the standardized TSST combined with a heat pain paradigm. The study demonstrates that (1) the psychosocial stress condition induced an objective endocrine HPA and ANS, as well as a subjective state anxiety stress response, and (2) the TSST produced a small but significant SIA response which (3) was not mediated by cortisol level, sAA activity, or state anxiety.

Changes of both objective neuroendocrine as well as subjective parameters indicated that our psychosocial stress condition induced the intended stress responses as evidenced by an increase of cortisol level, sAA activity, state anxiety score, and cognitive anticipatory stress appraisal, while these reactions were absent in the control condition. Interestingly and contrarily to previous observations [48], we did not observe a significant association between subjective and objective responses to stress in general and cognitive appraisal and cortisol in particular, which might be related to the somewhat lower cortisol responses observed in this study. Since there were no stress responses in the control condition, stress induction by the repeated pain assessment is unlikely. The repeated within-subject analysis shows a small, albeit significant, increase in heat pain tolerance after psychosocial stress but no effect of psychosocial stress on heat pain threshold. However, results for subjective pain ratings did not corroborate these differences; while intensity ratings slightly increased after the TSST, no such pre-post differences were found for subjective pain valence. A possible expla-

Psychosocial Stress-Induced Analgesia nation for this exclusive stress-induced tolerance responsiveness is that pain threshold and tolerance are supposed to capture different aspects of pain. While the pain threshold assessment indicates primary sensory discrimination of nociceptive quality, pain tolerance decisions reflect an unwillingness to receive more intense stimuli [59] and are thought to measure more psychological features [60]. Although tolerance limit is also a sensory decision, it is generally considered as more responsive to affective, motivational, and cognitive influences than pain threshold. Also, we cannot rule out that our reported small difference between threshold and tolerance following the stress induction might be attributable to the 2 different pain assessment methods rather than to a fundamental stress-sensitive difference between these 2 pain measures.

Contrary to the findings of al'Absi et al. $[6,30]$, who showed elevated cortisol levels following a brief pain stimulus (90 s), we could not see any biological or subjective response to the employed heat pain stimuli. Several reasons, such as the type of the stressor and the time course of the measurement, may account for these differences. Neither objective heat pain threshold or tolerance nor subjective pain intensity or valence correlated with cortisol level, sAA activity, or state anxiety. Thus, with regard to subjective heat perception, we could not replicate the recent findings of demonstrating a negative association between cortisol and pain intensity in men [30] or of positive correlations between sAA activity and intensity as well as unpleasantness ratings in response to acute heat pain stimuli [37]. Furthermore, mediation analysis revealed that SIA was not mediated by cortisol level, sAA activity, or subjective state anxiety. Similarly to the findings of al'Absi and Petersen [6], the stress-associated rise in cortisol concentrations was not responsible for pain relief in the stress condition. Given that complex context-dependent SIA processes are known to produce many generalized effects, multiple physiological actions such as monoaminergic mediators may contribute to anti-nociceptive effects and to pain [23]. In particular, it is possible that other hormones not assessed in the present investigation and that have all been implicated in pain regulation, such as $\mathrm{CRH}, \mathrm{ACTH}$, and $\beta$-endorphin [61-63], may contribute to the reduced pain sensitivity under acute laboratory stress [6]. However, and with regard to the lack of any association between pain and stress-induced biological responses, we need to acknowledge that the relatively small and specific analgesic response in our study might have precluded the detection of a possible relationship between these parameters.

Neuropsychobiology 2016;74:87-95 DOI: $10.1159 / 000454986$ 
Although subjective state STAI anxiety was highest before the TSST, it did not serve as a mediator of SIA. The influence of anxiety in pain perception has been described as 2-fold. If anxiety is not referred to the noxious stimuli it can cause pain relief, while the other way around people experience even more pain [64]. Context-induced negative expectations about the experiment and what will occur during the study might induce anticipatory anxiety, which is known to trigger 2 independent processes, hyperactivity in the HPA axis on the one hand and activation of cholecystokininergic pro-nociceptive systems on the other hand [65], and it is possible that the activation of the cholecystokinin system might have interacted and even dampened the strength of the SIA response.

We cannot rule out that the observed SIA was simply too small to detect the important inter-subject variability of complex SIA processes. Also, it is possible that the elicited analgesia following the TSST might be the result of distracting attention away from the source of pain [66]. It would thus be interesting to assess possible analgesic effects of exogenous glucocorticoid administration to levels comparable with those observed in our study, but without the possible distraction by the psychosocial stress paradigm.

Clearly, the generalizability and potential implications to clinical practice of our data are limited because our SIA responses were - albeit statically significant - numerically very small, and our study was designed to investigate the role of experimentally induced psychosocial stress in acute, controllable thermal pain in healthy participants. Furthermore, it needs to be taken into account that we induced pain experimentally, which might be different to and involve different processes than pathological pain. However, our comprehensive methodological and statistical approach may help to better differentiate between multiple forms of complex and heterogeneous SIA processes and their different mediators in the context of neurocognitiveaffective pain research. An improved understanding of the neurobiological mechanisms of SIA has the potential to elucidate the complex interplay between nociceptive processing and stress as well as to identify potential new therapeutic treatments for stress and pain-related disorders such as post-traumatic stress disorder [67], borderline personality disorder [45], and chronic pain syndromes [68].

\section{Disclosure Statement}

The authors certify that they have no affiliations with or involvement in any organization or entity with any financial or nonfinancial interest in the subject matter or materials discussed in this paper.

\section{References}

1 Amit Z, Galina ZH: Stress-induced analgesia: adaptive pain suppression. Physiol Rev 1986; 66:1091-1120.

2 Parikh D, Hamid A, Friedman TC, Nguyen K, Tseng A, Marquez P, Lutfy K: Stress-induced analgesia and endogenous opioid peptides: the importance of stress duration. Eur J Pharmacol 2011;650:563-567.

3 Willer JC, Dehen H, Cambier J: Stress-induced analgesia in humans: endogenous opioids and naloxone-reversible depression of pain reflexes. Science 1981;212:689-691.

4 Flor H, Grüsser SM: Conditioned stress-induced analgesia in humans. Eur J Pain 1999; 3:317-324.

5 Flor H, Birbaumer N, Schulz R, Grüsser SM, Mucha RF: Pavlovian conditioning of opioid and nonopioid pain inhibitory mechanisms in humans. Eur J Pain 2002;6:395-402.

6 al'Absi M, Petersen KL: Blood pressure but not cortisol mediates stress effects on subsequent pain perception in healthy men and women. Pain 2003;106:285-295.

7 Yilmaz P, Diers M, Diener S, Rance M, Wessa M, Flor H: Brain correlates of stress-induced analgesia. Pain 2010;151:522-529.
8 al'Absi M, Nakajima M, Grabowski J: Stress response dysregulation and stress-induced analgesia in nicotine dependent men and women. Biol Psychol 2013;93:1-8.

9 Vachon-Presseau E, Martel MO, Roy M, Caron E, Albouy G, Marin MF, Plante I, Sullivan MJ, Lupien SJ, Rainville P: Acute stress contributes to individual differences in pain and pain-related brain activity in healthy and chronic pain patients. J Neurosci 2013;33: 6826-6833.

10 Janal MN, Colt EW, Clark WC, Glusman M: Pain sensitivity, mood and plasma endocrine levels in man following long-distance running: effects of naloxone. Pain 1984;19:13-25.

11 Lautenschläger G, Habig K, Best C, Kaps M, Elam M, Birklein F, Krämer HH: The impact of baroreflex function on endogenous pain control: a microneurography study. Eur J Neurosci 2015;42:2996-3003.

12 Rhudy JL, Meagher MW: Fear and anxiety: divergent effects on human pain thresholds. Pain 2000;84:65-75.

13 de Wied M, Verbaten MN: Affective pictures processing, attention, and pain tolerance. Pain 2001;90:163-172.
14 Rhudy JL, Meagher MW: Negative affect: effects on an evaluative measure of human pain. Pain 2003; 104:617-626.

15 Ford GK, Finn DP: Clinical correlates of stress-induced analgesia: evidence from pharmacological studies. Pain 2008;140:3-7.

16 Rhudy JL, Williams AE, McCabe KM, Russell JL, Maynard LJ: Emotional control of nociceptive reactions (ECON): do affective valence and arousal play a role? Pain 2008;136:250-261.

17 Wiech K, Tracey I: The influence of negative emotions on pain: behavioral effects and neural mechanisms. Neuroimage 2009;47:987-994.

18 Schedlowski M, Enck P, Rief W, Bingel U: Neuro-bio-behavioral mechanisms of placebo and nocebo responses: implications for clinical trials and clinical practice. Pharmacol Rev 2015;67:697-730.

19 Quelhas Martins A, McIntyre D, Ring C: Aversive event unpredictability causes stressinduced hypoalgesia. Psychophysiology 2015; 52:1066-1070.

20 Fechir M, Breimhorst M, Kritzmann S, Geber C, Schlereth T, Baier B, Birklein, F: Naloxone inhibits not only stress-induced analgesia but also sympathetic activation and baroreceptorreflex sensitivity. Eur J Pain 2012;16:82-92. 
21 Millan MJ: Descending control of pain. Prog Neurobiol 2002;66:355-474.

22 Schlereth T, Birklein F: The sympathetic nervous system and pain. Neuromolecular Med 2008; 10:141-147.

23 Butler RK, Finn DP: Stress-induced analgesia. Prog Neurobiol 2009;88:184-202.

24 Terman GW, Lewis JW, Liebeskind JC: The effects of corticosterone on opioid stress analgesia. Proc West Pharmacol Soc 1984;27:447450.

25 Song ZH, Takemori AE: Involvement of spinal kappa opioid receptors in the antinociception produced by intrathecally administered corticotropin-releasing factor in mice. J Pharmacol Exp Ther 1990;254:363-368.

26 Sutton LC, Fleshner M, Mazzeo R, Maier SF, Watkins LR: A permissive role of corticosterone in an opioid form of stress-induced analgesia: blockade of opiate analgesia is not due to stress-induced hormone release. Brain Res 1994;663;19-29.

27 Filaretov AA, Bogdanov AI, Yarushkina NI Stress-induced analgesia. The role of hormones produced by the hypophyseal-adrenocortical system. Neurosci Behav Physiol 1996 26:572-578.

28 Lariviere WR, Melzack R: The role of corticotropin-releasing factor in pain and analgesia. Pain 2000;84:1-12.

29 Finn DP, Jhaveri MD, Beckett SRG, Madjd A, Kendall DA, Marsden CA, Chapman V: Behavioral, central monoaminergic and hypothalamo-pituitary-adrenal axis correlates of fear-conditioned analgesia in rats. Neuroscience 2006;138:1309-1317.

30 al'Absi M, Petersen KL, Wittmers LE: Adrenocortical and hemodynamic predictors of pain perception in men and women. Pain 2002;96:197-204.

31 Benarroch EE: Pain-autonomic interactions. Neurol Sci 2006;27(suppl 2):S130-S133.

32 Bantel C, Trapp S: The role of the autonomic nervous system in acute surgical pain processing - what do we know? Anaesthesia 2011;66 541-544.

33 Maixner W, Touw KB, Brody MJ, Gebhart GF, Long JP: Factors influencing the altered pain perception in the spontaneously hypertensive rat. Brain Res 1982;237:137-145.

34 al'Absi M, Buchanan T, Lovallo WR: Pain perception and cardiovascular responses in men with positive parental history for hypertension. Psychophysiology 1996;33:655-661.

35 Fillingim RB, Maixner W: The influence of resting blood pressure and gender on pain responses. Psychosom Med 1996;58:326-332.

36 van Stegeren AH, Wolf OT, Kindt M: Salivary alpha amylase and cortisol responses to different stress tasks: impact of sex. Int J Psychophysiol 2008;69:33-40.

37 Wittwer A, Krummenacher P, La Marca R, Ehlert U, Folkers G: Salivary alpha-amylase correlates with subjective heat pain perception. Pain Med 2016;17:1131-1136.

38 Kirschbaum C, Pirke KM, Hellhammer DH: The 'Trier Social Stress Test' - a tool for inves- tigating psychobiological stress responses in a laboratory setting. Neuropsychobiology 1993;28:76-81.

39 Krummenacher P, Candia V, Folkers G Schedlowski M, Schönbächler G: Prefrontal cortex modulates placebo analgesia. Pain 2010;148:368-374.

40 Pud D, Golan Y, Pesta R: Hand dominancy - a feature affecting sensitivity to pain. Neurosci Lett 2009;467:237-240.

41 Kirschbaum C, Kudielka BM, Gaab J, Schommer NC, Hellhammer DH: Impact of gender, menstrual cycle phase, and oral contraceptives on the activity of the hypothalamus-pituitary-adrenal axis. Psychosom Med 1999; 61:154-162.

42 Kirschbaum C, Wüst S, Strasburger CJ: 'Normal' cigarette smoking increases free cortisol in habitual smokers. Life Sci 1992;50:435442.

43 Badrick E, Bobak M, Britton A, Kirschbaum C, Marmot M, Kumari M: The relationship between alcohol consumption and cortisol secretion in an aging cohort. J Clin Endocrinol Metab 2008;93:750-757.

44 Laakmann G, Wittmann M, Gugath M, Mueller OA, Treusch J, Wahlster U, Stalla GK: Effects of psychotropic drugs (desimipramine, chlorimipramine, sulpiride and diazepam) on the human HPA axis. Psychopharmacology (Berl) 1984;84:66-70.

45 Ludäscher P, Valerius G, Stiglmayr C, Mauchnik J, Lanius RA, Bohus M, Schmahl C: Pain sensitivity and neural processing during dissociative states in patients with borderline personality disorder with and without comorbid posttraumatic stress disorder: a pilot study. J Psychiatry Neurosci 2010;35:177-184.

46 Stanley B, Sher L, Wilson S, Ekman R, Huang YY, Mann JJ: Non-suicidal self-injurious behavior, endogenous opioids and monoamine neurotransmitters. J Affect Disord 2010;124: 134-140.

47 Allen AP, Kennedy PJ, Cryan JF, Dinan TG, Clarke G: Biological and psychological markers of stress in humans: focus on the Trier Social Stress Test. Neurosci Biobehav Rev 2014; 38:94-124.

48 Gaab J, Rohleder N, Nater UM, Ehlert U: Psychological determinants of the cortisol stress response: the role of anticipatory cognitive appraisal. Psychoneuroendocrinology 2005; 30:599-610.

49 Perogamvros I, Owen LJ, Newell-Price J, Ray DW, Trainer PJ, Keevil BG: Simultaneous measurement of cortisol and cortisone in human saliva using liquid chromatography-tandem mass spectrometry: application in basal and stimulated conditions. J Chromatogr B Analyt Technol Biomed Life Sci 2009;877: 3771-3775.

50 Faul F, Erdfelder E, Buchner A, Lang A-G: Statistical power analyses using $\mathrm{G}^{*}$ Power 3.1: tests for correlation and regression analyses. Behav Res Methods 2009;41:1149-1160.

51 Pruessner JC, Kirschbaum C, Meinlschmid G, Hellhammer DH: Two formulas for compu- tation of the area under the curve represent measures of total hormone concentration versus time-dependent change. Psychoneuroendocrinology 2003;28:916-931.

52 Pinheiro JC, Bates DM: Mixed-Effects Models in S and S-PLUS. New York, Springer, 2002.

53 Singer JD, Willett JB: Applied Longitudinal Data Analysis: Modeling Change and Event Occurrence. New York, Oxford University Press, 2003

54 Kenny DA, Korchmaros JD, Bolger N: Lower level mediation in multilevel models. Psychol Methods 2003;8:115-128.

55 Baron RM, Kenny DA: The moderator-mediator variable distinction in social psychological research: conceptual, strategic, and statistical considerations. J Pers Soc Psychol 1986; 51:1173-1182.

56 MacKinnon DP: Introduction to Statistical Mediation Analysis. New York, Erlbaum, 2008.

57 MacKinnon DP, Fairchild AJ, Fritz MS: Mediation analysis. Annu Rev Psychol 2007;58: 593-614.

58 MacKinnon DP, Krull JL, Lockwood CM Equivalence of the mediation, confounding and suppression effect. Prev Sci 2000;1:173181.

59 Harris G, Rollman GB: The validity of experimental pain measures. Pain 1983;17:369-376.

60 Merskey H, Spear FG: Pain - Psychological and Psychiatric Aspects. London, Baillière, Tindall \& Cassell, 1967.

61 Hartwig AC: Peripheral beta-endorphin and pain modulation. Anesth Prog 1991;38:7578.

62 Yarushkina NI: The role of hypothalamo-hypophyseal-adrenocortical system hormones in controlling pain sensitivity. Neurosci Behav Physiol 2008;38:759-766.

63 Yarushkina NI, Bagaeva TR, Filaretova LP: Central corticotropin-releasing factor (CRF) may attenuate somatic pain sensitivity through involvement of glucocorticoids. J Physiol Pharmacol 2011;62:541-548.

64 al'Absi M, Rokke PD: Can anxiety help us tolerate pain? Pain 1991;46:43-51.

65 Benedetti F, Amanzio M, Vighetti S, Asteggiano G: The biochemical and neuroendocrine bases of the hyperalgesic nocebo effect. J Neurosci 2006;26:12014-12022.

66 Peyron R, García-Larrea L, Grégoire MC, Costes N, Convers P, Lavenne F, Mauguière F, Michel D, Laurent B: Haemodynamic brain responses to acute pain in humans: sensory and attentional networks. Brain 1999;122: $1765-1780$.

67 Geuze E, Westenberg HG, Jochims A, de Kloet CS, Bohus M, Vermetten E, Schmahl C: Altered pain processing in veterans with posttraumatic stress disorder. Arch Gen Psychiatry 2007;64:76-85.

68 Blackburn-Munro G, Blackburn-Munro RE: Chronic pain, chronic stress and depression: coincidence or consequence? J Neuroendocrinol 2001;13:1009-1023. 